\section{MDSORT: A special-purpose multidimensional scaling program for sorting data}

\author{
YOSHIO TAKANE \\ McGill University, Montreal, Quebec H3A IB1, Canada
}

MDSORT (Takane, 1980) is a multidimensional scaling (MDS) program specifically designed for sorting data. In the stimulus sorting method, the subjects are asked to sort a set of stimuli into as many groups (clusters) as they wish so that the stimuli in a cluster are more similar to each other than those in different clusters. This method has enjoyed great popularity among social scientists as a quick and easy data collection method for similarities. The sorting task is easy to perform, so the method is particularly suitable when one has naive subjects and/or a large number of stimuli (up to 100) and when it is difficult to obtain direct (dis)similarity judgments for every possible pair of stimuli. Typically, the sorting data are analyzed by nonmetric MDS using such procedures as KYST (Kruskal \& Wish, 1978), SSA (Lingoes, 1973), and ALSCAL (Takane, Young, \& de Leeuw, 1977). However, in order to apply nonmetric MDS, the sorting data have to be converted into similarity data. The analysis results are typically dependent on the particular similarity index used. The following is a brief description of an MDS program that simultaneously converts the original sorting data into similarity data and finds a representation of the similarity data based on a single common criterion.

Description. MDSORT allows a user to directly input original sorting data. The program then finds a similarity matrix and obtains a configuration of stimulus points in a multidimensional euclidean space in such a way that the sum of squared intercluster distances averaged over the subjects is a maximum under suitable normalization restrictions on the configuration. Due to a unique feature of the method detailed in Takane (1980), stimulus coordinates in a lower dimensional solution remain intact in a higher dimensional solution. (For example, the first two dimensions in a three-dimensional solution are the same as those in a two-dimensional solution.) A chi-square test for appropriate dimensionality of the configuration is also available. Cluster centroids for the stimuli classified into certain sorting clusters may be optionally calculated for each subject and used as information about the individual differences in sorting behavior. This information was unavailable with the conventional MDS procedures.

Input. Input to the program consists of several job parameters (title, number of stimuli, subjects and

The preparation of this manuscript was supported by Grant A6394 to the author by the Natural Sciences and Engineering Research Council of Canada. dimensions, input data format, and input/output options) and the data for $\mathrm{N}$ subjects, all punched on cards in a specific format. For each subject, the sorting data indicate, by consecutive integers starting from 1 , cluster memberships of stimuli. Suppose there are five stimuli classified into three groups by a certain subject, one group with Stimuli 1 and 4, another with Stimuli 3 and 5 , and a third with Stimulus 2 by itself. Then the data for the subject should be "1 32212 ." Cluster numbers may be arbitrary (i.e., it does not matter which clusters may be designated by which integers), however, provided that we use the first $\mathrm{N}_{k}$ positive integers when the number of clusters for the subject is $\mathrm{N}_{\mathbf{k}}$. Missing data may be handled by setting up additional clusters, each consisting of each stimulus corresponding to a missing observation.

Output. The program prints out the value of the chi-square statistic and the associated degrees of freedom for each dimensionality (from which one can determine appropriate dimensionality in the representation space) and the stimulus coordinates in as many dimensions as specified on a job parameter card. They may optionally be plotted and/or punched out on cards. Cluster centroids may also be calculated, printed out, plotted, and/or punched out.

Language and Computer. MDSORT is written in FORTRAN IV for use on batch processing system with an ordinary card reader and a line printer.

Availability. A copy of the program and the program users' manual (Takane, Note 1), which includes sample input and output, may be obtained by writing to Yoshio Takane, Department of Psychology, McGill University, 1205 Avenue Docteur Penfield, Montreal, Quebec H3A 1B1, Canada. Requests should be accompanied by a nine-track magnetic tape and a $\$ 5$ check (made payable to McGill University) to cover the computer cost, photocopying cost, and postage.

\section{REFERENCE NOTE}

1. Takane, Y. How to use MDSORT: A special purpose multidimensional scaling program for sorting data. Unpublished user's manual, McGill University, 1981.

\section{REFERENCES}

Kruskal, J. B., \& Wish, M. Multidimensional scaling (Sage University Paper Series 07-001). Beverly Hills, Calif: Sage Publications, 1978.

Lingoes, J. C. The Guttman-Lingoes nonmetric program series. Ann Arbor, Mich: Mathesis Press, 1973.

TAKane, Y. Analysis of categorizing behavior by a quantification method. Behaviormetrika, 1980, 8, 57-67.

Takane, Y., Young, F. W., \& de Leeuw, J. Nonmetric individual differences multidimensional scaling: An alternating least squares method with optimal scaling features. Psychometrika, 1977, 42, 7-67.

(Accepted for publication April 28, 1981.) 